\title{
Ultrafast Excited-State Dynamics in Biological Environments
}

\author{
Alexandre Fürstenberg and Eric Vauthey*
}

\begin{abstract}
We discuss and illustrate by several examples how the ultrafast excited-state dynamics of a chromophore can be altered when changing its environment from a homogenous solution to a biological molecule such as proteins or nucleic acids.
\end{abstract}

Keywords: Excited-state quenching · Fluorescence polarization anisotropy · Fluorescent probes Solvation dynamics - Vibrational energy relaxation

\section{Introduction}

Fluorescence spectroscopy has become a widespread tool in chemistry, biology, and medicine. Its popularity arises from its high sensitivity and from the fact that the optical properties of many fluorescent dyes in the condensed phase are strongly influenced by their direct chemical environment (liquid solution, protein or DNA environment, thin film, etc.).

Many applications as diverse as optoelectronic devices, molecular switches, chemosensors and other diagnostic or detection tools rely on static chromophore-environment interactions, which affect properties such as the transition energy or the molecular geometry. However, the environment of the chromophore can also affect its excited-state dynamics.

For example, relaxation processes such as medium reorganization (solvation) and

${ }^{*}$ Correspondence: Prof. Dr. E. Vauthey

University of Geneva

Department of Physical Chemistry

Quai Ernest-Ansermet 30

$\mathrm{CH}-1211$ Genève 4

Tel.: +4122379 6804

Fax: + 41223796518

E-Mail: eric.vauthey@chiphy.unige.ch vibrational cooling, which are usually ultrafast and occur directly after optical generation of the excited-state population are known to be largely determined by the nature of the direct environment of the chromophore. [1] Photochemical processes, such as electron ${ }^{[2]}$ and proton transfer ${ }^{[3]}$ or isomerization via large amplitude motion, $\left.{ }^{4}\right]$ which take place on a time scale of a few tens of femtoseconds up to nanoseconds are also strongly influenced by the environment. Therefore, investigation of the excited-state dynamics of chromophores in a biological environment may yield structural information on the biological system. Biological systems studied by femtosecond-resolved techniques are usually interrogated via intrinsic reporter groups such as single tryptophan residues, ${ }^{[5]}$ the chromophore of GFP, ${ }^{[6]}$ or cofactors such as retinal or heme moieties. ${ }^{[7,8]}$ As long as they do not impair the biological function, external, well-characterized, and well-positioned fluorophores should also allow the local environment of systems, which cannot be intrinsically photoactivated, to be probed if they are chosen to be sensitive to a local parameter (polarity, chirality). We will illustrate here how the interaction between external probes of this kind and given biomolecular systems (protein, DNA) affects ultrafast processes such as the decay of the fluorescence polarization anisotropy, inter- and intramolecular quenching, solvent dynamics, and vibrational energy relaxation.

\section{Fluorescence Polarization Anisotropy (FPA)}

A dye bound to a large biological molecule has often a reduced mobility compared to the free dye in a liquid. This mobility can be probed by monitoring the FPA decay,

$$
r(t)=\left[I_{\|}(t)-I_{\perp}(t)\right] /\left[I_{\|}(t)+2 I_{\perp}(t)\right]
$$

where $I_{\| \mid}(t)$ and $I_{1}(t)$ are the polarization components of the fluorescence intensity parallel, resp. perpendicular, to the polarization of the excitation light. Fig. 1A shows the FPA decay of the probe Lucifer Yellow (LY) tagged to biotin via a 3-atom (LY-3btn) and a 13-atom spacer (LY-13-btn), free in solution, and bound to avidin (Scheme). Avidin (Avd) and streptavidin are two homologous proteins particularly well suited for studying the influence of a protein environment on the fluorescence dynamics of extrinsic tags. These tetrameric proteins bind very tightly but non-covalently to up to four molecules of D-biotin (vitamin H), with the highest binding constant known in nature between a protein and its ligand, and biotin can be easily derivatised by a large variety of molecules and stay available for tight binding to the two proteins. ${ }^{[9]}$ The (strept)avidin-biotin system thus behaves as a tuneable 'molecular Velcro' and has been widely used in applications such as purifications, immunoassays, immobilization kits, diagnostic tools, cancer therapy, and recently also in efficient enantioselective catalysis. ${ }^{[10,11]}$ The FPA of free LY-3btn and LY-13-btn decays to zero with a time constant of the order of 150-200 ps, in good agreement with the Stokes-EinsteinDebye model for the reorientational diffusion of molecules of this size in water.[12] On the other hand, the decay of the FPA of LY-3-btn and LY-13-btn bound to avidin does not decay to zero, pointing to a strong 


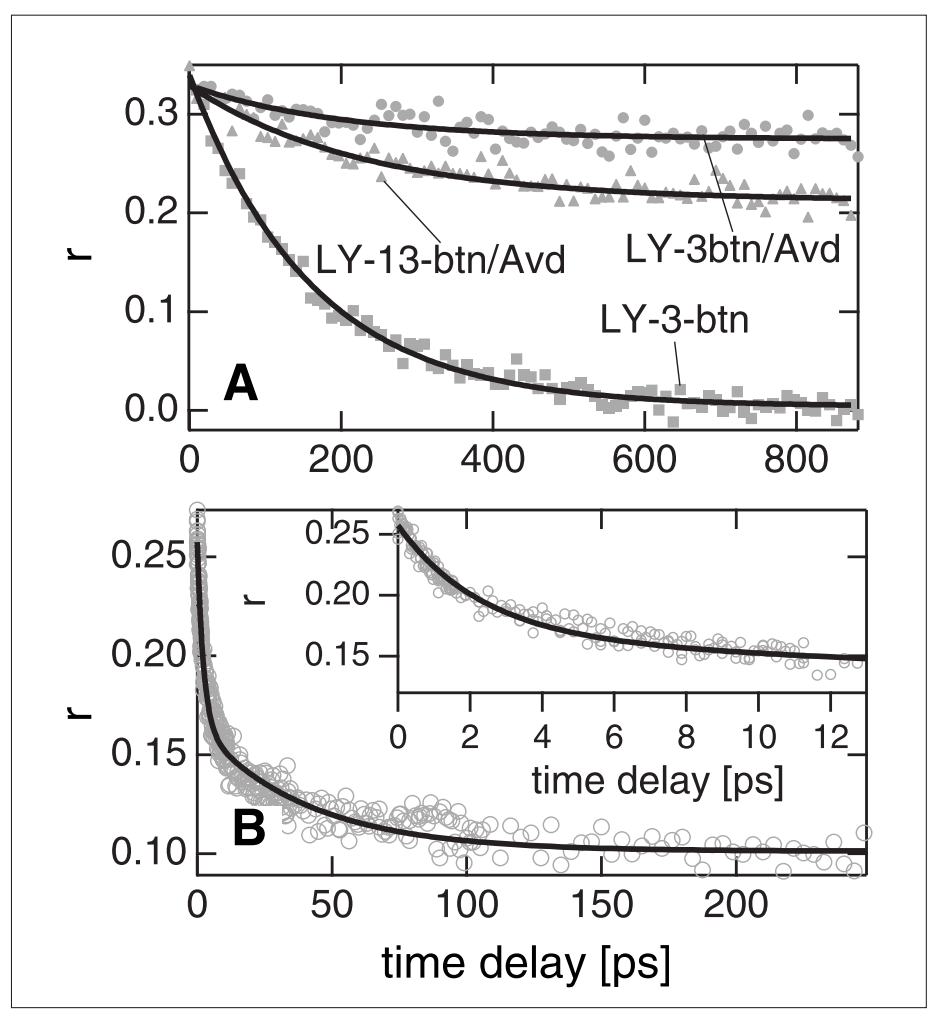

Fig. 1. A) Decay of the fluorescence anisotropy of LY-3-btn and LY-13btn in aqueous buffer solution and/or in avidin. B) Decay of the fluorescence anisotropy of YOYO-1 bound to DNA.

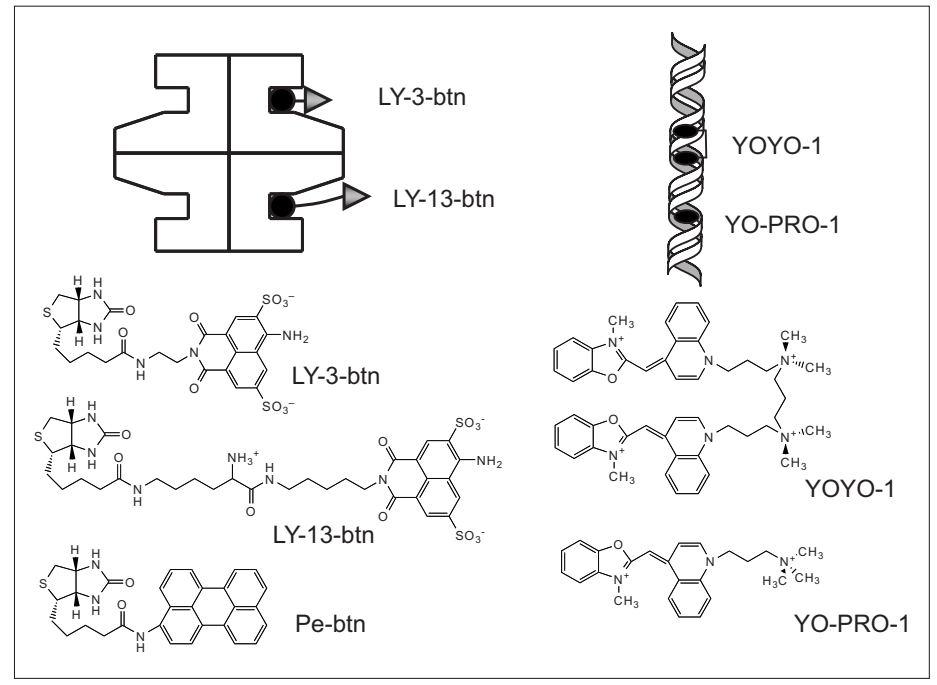

Scheme. (Left) Schematic representation of a construct between the fluorescent probe molecule Lucifer Yellow (triangle) and biotin (black circle) via a short or a long spacer and bound to tetrameric avidin. (Right) Schematic representation of the bisintercalator YOYO-1 or the simple intercalator $\mathrm{YO}-\mathrm{PRO}-1$ bound to DNA.

reduction of the mobility. With LY-3-btn, the chromophore is located rather closely to the binding pocket and experiences a more constrained environment than the chromophore in LY-13-btn. This explains its larger residual anisotropy compared to LY-13-btn.

In some cases, a more constrained environment upon binding to a large biomolecule does not result in a retardation of the FPA decay. Fig. 1B shows the example of the homodimeric Oxazole Yellow derivative YOYO-1,[13] which is known to bisintercalate into DNA ${ }^{[14]}$ (Scheme). The FPA decay is biphasic, with $2.6 \mathrm{ps}$ and $51 \mathrm{ps}$ components, while that of free YOYO- 1 in water occurs with a $\sim 60$ ps time constant, in agreement with reorientational diffusion. The FPA decay of YOYO-1 in DNA is clearly not due to rotational motion of the
DNA:dye complex, but can be ascribed to intramolecular excitation energy hopping between the two chromophoric units of the dye. ${ }^{[13]}$ As a consequence of DNA helicity, the transition dipoles moments of the two chromophoric units of YOYO-1 are not parallel but make a close to $90^{\circ}$ dihedral angle. This angle prevents strong excitonic coupling between the chromophores and induces a net reorientation of the emitting dipole moment upon energy hopping. The anisotropy decay can indeed be reproduced using Förster theory for energy transfer and assuming a Gaussian distribution of dihedral angles centered at $83^{\circ}$ with a full width at half-maximum of $10^{\circ} .{ }^{[13]}$ The FPA decay does not depend on the DNA:YOYO-1 ratio, i.e. on the distance between two dye molecules, confirming that the energy hopping is really intramolecular.
With the monomeric intercalator YOPRO-1, which is actually half the YOYO1 molecule (Scheme), the FPA decay was also found to be biexponential, with similar time scales as with YOYO-1. ${ }^{[15]}$ However, the amplitudes of the decay components strongly depend on the DNA:dye ratio since the energy hopping is intermolecular: the higher the loading in dye molecules of the DNA strand, the shorter the average dye-dye distance and the more efficient the energy hopping.[15]

\section{Inter- and Intramolecular Quenching}

The binding of a chromophore to a protein or a nucleic acid can be accompanied by changes in its fluorescence quantum yield. In most cases, this variation is associated with the enhancement or the inhibition of a quenching process and thus appears as a change in the fluorescence lifetime.

For example, the fluorescence quantum yield of LY-13-btn decreases from 0.23 to 0.14 upon binding to avidin. A 30 ps decay component observed only in the fluorescence decay of bound LY-13-btn can account for this reduction of quantum yield. Interestingly such fluorescence quenching is not observed with the shorter LY-3-btn. The fast quenching component most probably arises from electron transfer between residue Trp 110 of avidin and the dye. It has indeed been shown that LY can undergo efficient and ultrafast photoinduced electron transfer from tryptophan (Trp) in aqueous solution. ${ }^{[12]}$ With the longer spacer of LY13-btn, the dye can access a region of the protein surface where it can interact with the residue Trp110 and undergo electron transfer. On the other hand, such Trp residues are not present in the vicinity of the chromophore of LY-3-btn. Molecular dynamics simulations are currently being performed to confirm this hypothesis.

On the other hand, free YO-PRO-1 and YOYO-1 are virtually non-fluorescent in solution but their fluorescence quantum yield is as large as 0.45 when they are intercalated into DNA. ${ }^{[16]}$ The discovery of this huge fluorescence contrast between the free and bound forms of these dyes in the 1990s caused a revolution in molecular biology. Actually it allowed DNA to be detected for the first time at a sensitivity comparable to that of radioactive probes, but without the danger inherent in radioactivity. ${ }^{[17]}$ We have shown that two different mechanisms are responsible for this fluorescence enhancement upon DNA binding. ${ }^{[13,18,19]}$ In water, these dyes tend to form dimeric H-type aggregates, which are non-fluorescent because of excitonic interaction between the chromophoric units. ${ }^{[13]}$ On the other hand, the non-aggregated dyes undergo ultrafast 
non-radiative deactivation via an isomerization process involving a large-amplitude torsional motion, which takes place with a time constant of 3-6 ps depending on the dye structure (Fig. 2). Because of those two processes, these dyes are essentially nonfluorescent in water. Upon intercalation into DNA, the aggregates are disrupted and the strong excitonic interaction is removed. Interestingly, this suppression is not really caused by the distance between the chromophores in DNA but rather by their quasiorthogonal relative geometry. Moreover, once intercalated, the excited dyes can no longer undergo photoisomerization as the constricted environment inhibits large amplitude torsional motion. As a consequence, their excited state lifetime increases to several nanoseconds and they become strongly fluorescent (Fig. 2).

\section{Solvation Dynamics}

The binding of a chromophore to a biomolecule is not necessarily accompanied by a change in the stationary fluorescence. However, differences in the early fluorescence dynamics, due for example to the relaxation of a polar environment, can still be observed. As illustrated in Fig. 3, solvent relaxation around an excited chromophore experiencing a change in electric dipole moment is accompanied by a decrease of the $S_{1}-S_{0}$ energy gap and thus by a red shift of the fluorescence spectrum. The measurement of this time-dependent Stokes shift (TDSS) allows polar solvation dynamics to be investigated. [1]

Water plays a very important role in the equilibrium structure and the function of biological molecules such as nucleic acid and proteins. Over the past years, intense efforts have been invested in the study of the motion of water molecules at the interface with proteins and DNA. ${ }^{[5,20,21]}$ We have investigated the TDSS of the above-mentioned YOYO dyes in bulk water and in DNA. ${ }^{[13,18]}$ A TDSS time constant of about 1 ps was found with the homodimeric YOYO-1 bisintercalated into double-stranded DNA. As this time constant is similar to that for diffusional motion in bulk water, it is very tempting to attribute it to the diffusive motion of water molecules surrounding DNA, although the dye is partially shielded from water. However, intercalation of the YOYOdyes is known to partially unwind the DNA helix and might favor the access of water to the chromophores. ${ }^{[14]}$ The absence of any slower decay component suggests that there is no significant reorganization of the DNA interior upon excitation of the dyes, unless it takes place on a time scale very similar to that of water, or that the probe molecule is not sensitive enough to feel these effects. On the other hand, with the single inter-

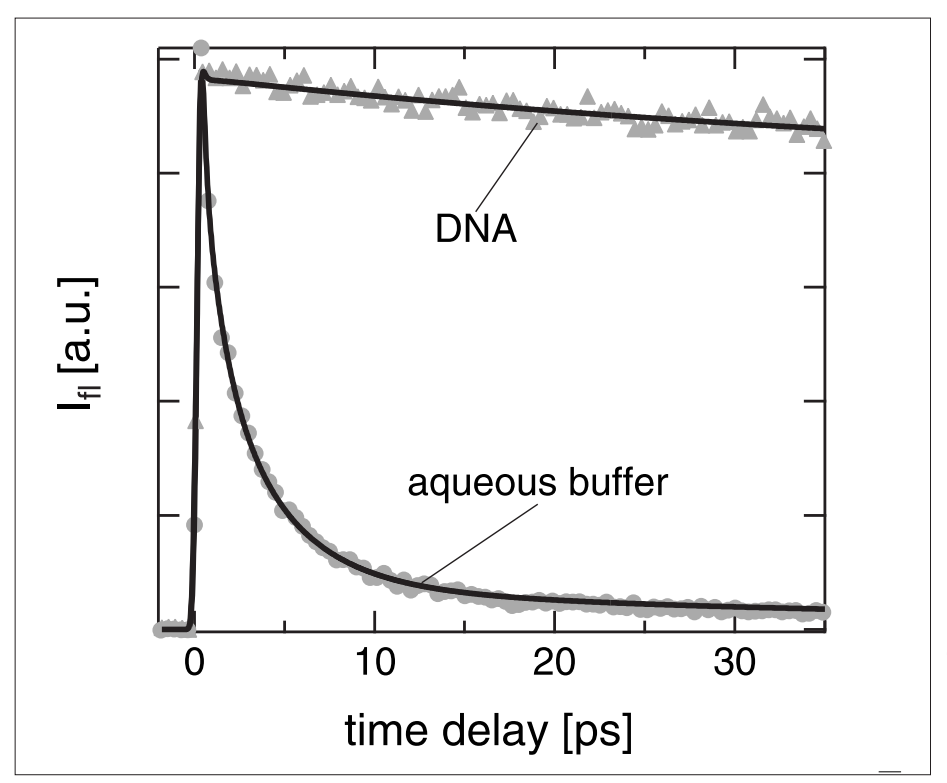

Fig. 2. Early fluorescence dynamics of YOYO-1 in aqueous buffer solution and bound to DNA

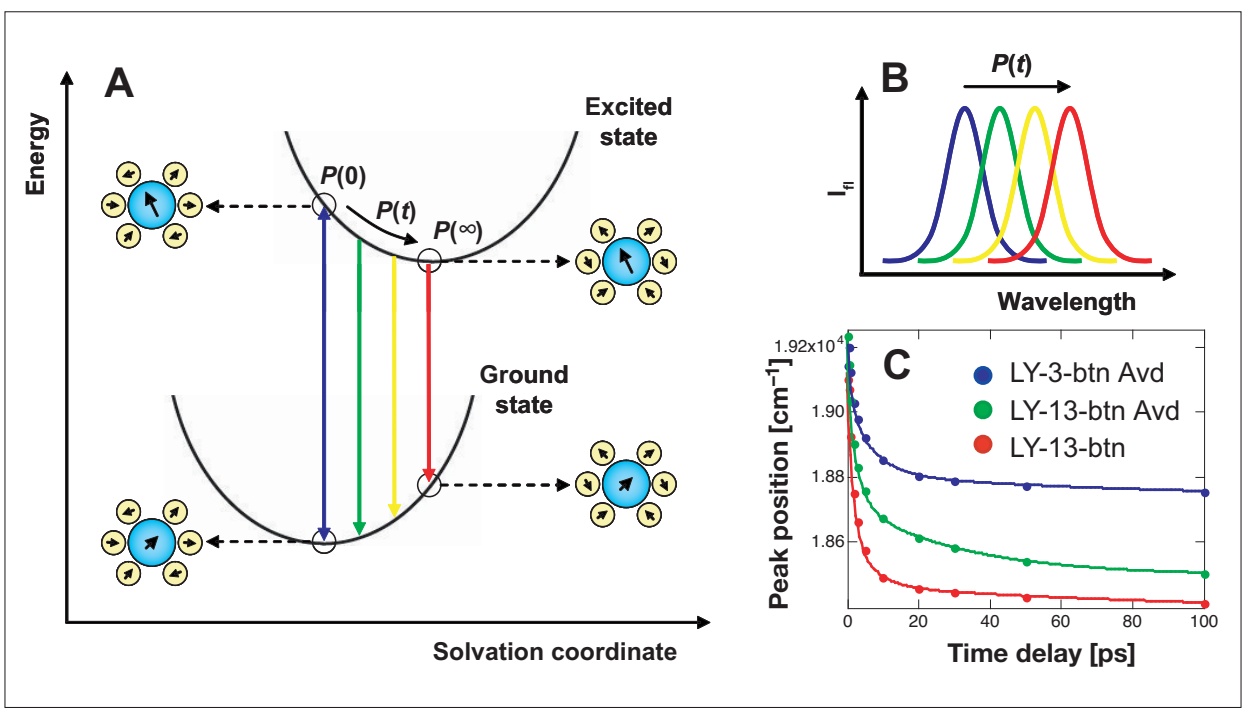

Fig. 3. A, B) Schematic representation of the time-dependent Stokes shift (TDSS). Right after optical excitation, the solvent is in a non-equilibrium configuration $(P(0))$. As it starts relaxing, the fluorescence spectrum shifts to the red $(P(t))$ until equilibrium is achieved $(P(\infty))$. C) TDSS of LY-3-btn and LY-13-btn in aqueous buffer solution and/or in avidin.

calator YO-PRO-1, a biphasic behavior of the TDSS was observed. with much lower amplitude than with YOYO-1 and with time constants of 2.3 and 41 ps. ${ }^{[15]}$ YO-PRO-1 intercalates between two base pairs and is thus expected to impose less pronounced geometrical constraints on the double helix and could therefore be more shielded from bulk water than the bisintercalator YOYO1. Such slowing down of TDSS dynamics at biomolecular interfaces had previously been observed by others ${ }^{[5]}$ but its origin is still debated.[20,21] The existence of a water layer 'bound' to the protein with much slower dynamics than that of bulk water has first been proposed, ${ }^{[5]}$ but simulations tend to show that the slower part of the dynamics originates from protein or coupled protein-solvent fluctuations ${ }^{[21,22]}$ and not only from a different population of water molecules. Our results show that the expo- sition of the probe to water can vary very strongly. A more definite assignment would be possible if the same fluorescent probe could be positioned at different locations on the biomolecule.

In order to better control the position of the fluorescent probe with respect to a biomolecular environment, we made use of the avidin-biotin system discussed above. LY3-btn and LY-13-btn have been specially designed to probe different environments of avidin. The above-mentioned differences in both polarization anisotropy decay and the excited state lifetime of LY-3-btn and LY-13-btn point precisely to different environments.

We found that the TDSS of the chromophore substantially slows down in the protein environment. The observed time constants are very similar for both the free and the bound conjugate ( $c a .1,5$, and 70 

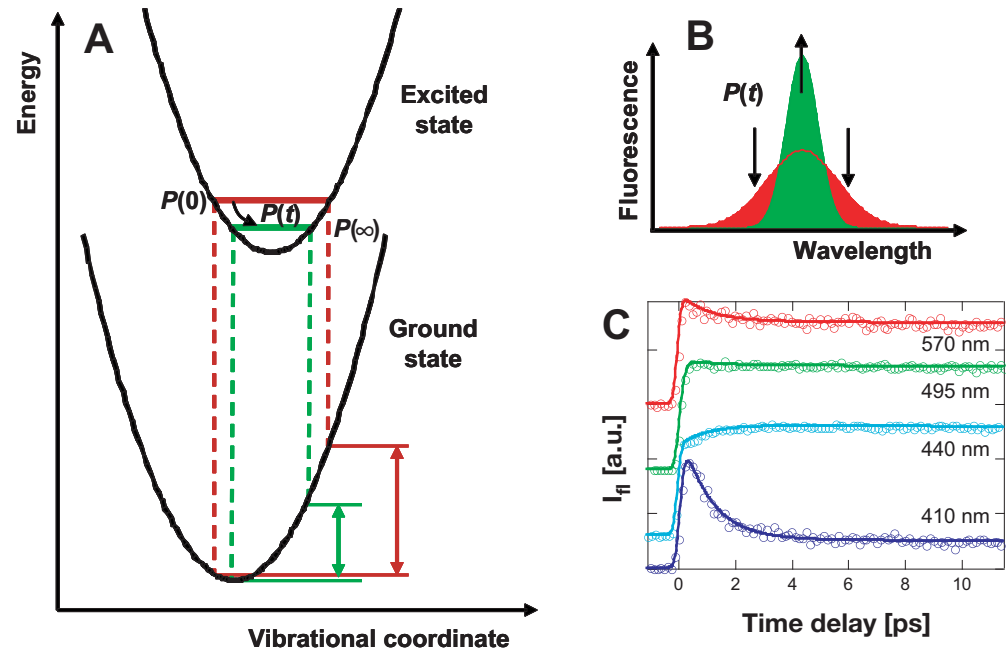

Fig. 4. A, B) Schematic representation of the effect of vibrational energy relaxation (VER) on the fluorescence spectrum. Just after optical excitation, the spectrum of the probe is broad (red spectrum, $P(0)$ ) and progressively narrows with time $(P(t))$ as VER takes place (green spectrum, $P(\infty)$ ). C) Early fluorescence dynamics of the $\mathrm{Pe}$-btn construct bound to avidin at different emission wavelengths.

ps), but the amplitude of the slower solvent relaxation components increases significantly upon binding to avidin (Fig. 3C). An intermediate behavior is observed with LY-13-btn linked to avidin, indicating that the nature of the environment of the chromophore is between those experienced by free LY-13-btn in water and by LY-3-btn in avidin. However, the existence of 'slow' moving water molecules could not be evidenced in these measurements.

\section{Vibrational Energy Relaxation}

Optical excitation of a molecule often does not populate the excited electronic state in its vibrational ground state $(0-0$ transition) but leads to the population of upper vibrational states. This so-called excess excitation energy is redistributed internally and dissipated to the environments through intramolecular vibrational redistribution (IVR) and vibration cooling (VC), respectively, to establish a thermal population of the various vibrational states. The details of these processes are not yet fully understood although recent studies have led to better insight into the role of the environment. ${ }^{[23]}$ Typical timescales for excited organic molecules in the condensed phase are tens of femtoseconds for the initial steps up to a dozen picoseconds depending on the solvent. ${ }^{[24]}$ The net effect of vibrational energy relaxation (VER) on the fluorescence dynamics is a narrowing of the fluorescence spectrum at quasi-constant excited-state population (Fig, 4A, B).

Perylene is a well-suited molecule for investigating VER dynamics since the early spectral dynamics of this centrosymmetric molecule does not involve solvent relax- ation. ${ }^{[24,25]}$ The early fluorescence dynamics of Pe-btn was investigated in presence of avidin. As shown in Fig. 4C, the decay of the fluorescence intensity at short and long wavelengths and the rise at intermediate wavelengths correspond to a spectral narrowing that can be assigned to VER. The main component of this narrowing has a lifetime of $1.5 \mathrm{ps}$, which turns out to be somewhat slower than for perylene in DMSO. ${ }^{[24]}$ Unfortunately neither perylene nor the Pe-btn conjugate are soluble enough in water to allow a comparison between the protein environment and bulk water to be drawn. Further experiments with perylene coupled to biotin via a longer, rigid spacer are planned in order to address this question. However, these preliminary results show that VER can be clearly monitored in a biomolecular environment.

\section{Conclusion}

We have shown that a biomolecular environment affects the early fluorescence dynamics of suitable fluorescent probes by influencing processes such as solvent dynamics, vibrational energy relaxation, excited-state quenching or the decay of the fluorescence polarization anisotropy. As these processes occur on an ultrafast timescale, femtosecond-resolved fluorescence spectroscopy is a powerful tool to gain insight on the nanoenvironment of fluorescent probes and then to obtain structural information on biomolecules.

\section{Acknowledgements}

The authors would like to thank Prof. T. Ward (University of Neuchâtel) and Prof. T. Deligeorgiev (Sofia University) for the continuous collaboration on the synthesis of fluorescent probes. This work was supported by the Fonds National Suisse de la Recherche Scientifique through project nr. 200020-115942.

Received: July 27, 2007

[1] R. M. Stratt, M. Maroncelli, J. Phys. Chem. 1996, 100, 12981.

[2] H. Heitele, Angew. Chem., Int. Ed. 1993, 32, 359 .

[3] N. Agmon, J. Phys. Chem. A 2005, 109, 13.

[4] E. Vauthey, Chem. Phys. 1995, 196, 569.

[5] S. K. Pal, A. H. Zewail, Chem. Rev. 2004, 104, 2099.

[6] D. Mandal, T. Tahara, S. R. Meech, $J$. Phys. Chem. B 2004, 108, 1102.

[7] F. Gai, K. C. Hasson, J. C. McDonald, P. A. Anfinrud, Science 1998, 279, 1886.

[8] M. H. Vos, J.-L. Martin, Biochim. Biophys. Acta 1999, 1411, 1.

[9] N. M. Green, Methods Enzymol. 1990, $184,51$.

[10] M. Wilchek, E. A. Bayer, 'Avidin-biotin technology', Academic Press, San Diego, 1990.

[11] C. M. Thomas, T. R. Ward, Chem. Soc. Rev. 2005, 34, 337.

[12] A. Fürstenberg, E. Vauthey, Photochem. Photobiol. Sci. 2005, 260.

[13] A. Fürstenberg, M. D. Julliard, T. G. Deligeorgiev, N. I. Gadjev, A. A. Vassilev, E. Vauthey, J. Am. Chem. Soc. 2006, 128, 7661.

[14] F. Johansen, J. P. Jacobsen, J. Biomol. Struct. Dyn. 1998, 16, 205.

[15] A. Fürstenberg, E. Vauthey, J. Phys. Chem. $B$ 2007, in print.

[16] H. S. Rye, M. A. Quesada, K. Peck, R. A. Mathies, A. N. Glazer, Nucleic Acids Res. 1991, 19, 327.

[17] P. Selvin, Science 1992, 257, 885 .

[18] A. Fürstenberg, M. D. Julliard, T. Deligeorgiev, N. Gadjev, A. A. Vassilev, E. Vauthey, in 'Femtochemistry VII: Fundamental Ultrafast Processes in Chemistry, Physics, and Biology', Eds. A. W. Castleman Jr, M. L. Kimble, Elsevier, Amsterdam, 2006.

[19] A. Fürstenberg, T. G. Deligeorgiev, N. I. Gadjev, A. A. Vasilev, E. Vauthey, Chem. Eur. J. 2007, in print.

[20] D. Andreatta, J. L. P. Lustres, S. A. Kovalenko, N. P. Ernsting, C. J. Murphy, R. S. Coleman, J. Am. Chem. Soc. 2005, 127, 7270.

[21] L. Nilsson, B. Halle, Proc. Nat. Acad. Sci. USA 2005, 102, 13867.

[22] A. A. Golosov, M. Karplus, J. Phys. Chem. B 2007, 111, 1482.

[23] C. G. Elles, F. F. Crim, Annu. Rev. Phys. Chem. 2006, 57, 273.

[24] A. Pigliucci, G. Duvanel, M. L. Lawson Daku, E. Vauthey, J. Phys. Chem. A 2007, 111, 6135 .

[25] A. Pigliucci, E. Vauthey, Chimia 2003, 57, 200. 